\title{
Antimicrobial activity of endophytic fungi from olive tree leaves
}

\author{
Cynthia Malhadas ${ }^{1} \cdot$ Ricardo Malheiro $^{1} \cdot$ José Alberto Pereira $^{1} \cdot$ \\ Paula Guedes de Pinho ${ }^{2} \cdot$ Paula Baptista $^{1}$
}

Received: 4 December 2016 / Accepted: 18 January 2017 / Published online: 6 February 2017

(C) Springer Science+Business Media Dordrecht 2017

\begin{abstract}
In this study, the antimicrobial potential of three fungal endophytes from leaves of Olea europaea $\mathrm{L}$. was evaluated and the host plant extract effect in the antimicrobial activity was examined. The volatile compounds produced by endophytes were identified by GC/MS and further correlated with the antimicrobial activity. In potato dextrose agar, both Penicillium commune and Penicillium canescens were the most effective inhibiting Gram-positive and -negative bacteria (up to 2.7-fold compared to $30 \mu \mathrm{g} /$ $\mathrm{mL}$ chloramphenicol), whereas Alternaria alternata was most effective inhibiting yeasts (up to 8.0-fold compared to $25 \mu \mathrm{g} / \mathrm{mL}$ fluconazole). The presence of aqueous leaf extract in culture medium showed to induce or repress the antimicrobial activity, depending on the endophytic
\end{abstract}

species. In the next step, various organic extracts from both A. alternata mycelium and cultured broth were prepared; being ethyl acetate extracts displayed the widest spectrum of anti-microorganisms at a minimum inhibitory concentration $\leq 0.095 \mathrm{mg} / \mathrm{mL}$. The volatile composition of the fungi that displayed the highest (A. alternata) and the lowest $(P$. canescens) antimicrobial activity against yeasts revealed the presence of six volatiles, being the most abundant components (3-methyl-1-butanol and phenylethyl alcohol) ascribed with antimicrobial potentialities. Overall the results highlighted for the first time the antimicrobial potential of endophytic fungi from $O$. europaea and the possibility to be exploited for their antimicrobial agents.

Electronic supplementary material The online version of this article (doi:10.1007/s11274-017-2216-7) contains supplementary material, which is available to authorized users.

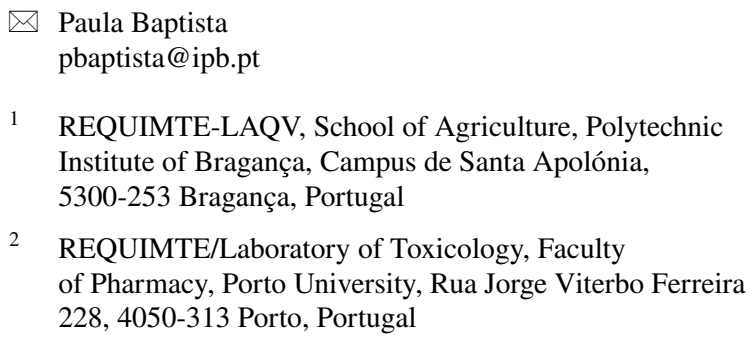




\section{Graphical Abstract}

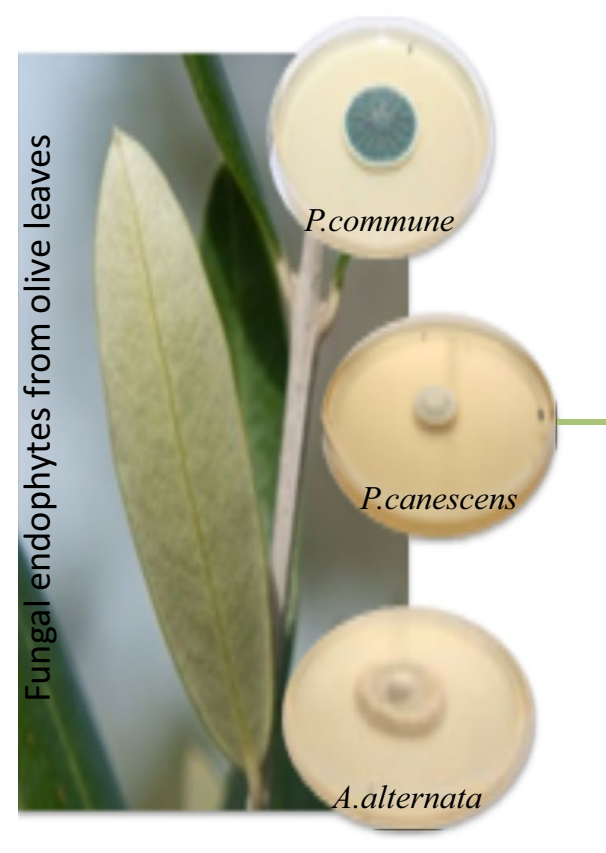

Keywords Alternaria alternata $\cdot$ Minimum inhibitory concentration · Olea europaea L. · Volatile fraction

\section{Introduction}

The search for natural bioactive compounds with application in the treatment and/or prevention of human diseases (Donadio et al. 2010) as well as in the design of nutraceuticals and functional food products (Gil-Chávez et al. 2013) has increased markedly over the last few years. Endophytic fungi are one of the most prominent producers of novel natural products (Selim et al. 2012). These fungi are characterized to colonize the living internal tissues of all higher plants without causing any damage to the host (Hyde and Soytong 2008). In this relationship endophytes produce a broad variety of bioactive secondary metabolites that can affect host plant physiology, defense and tolerance against biotic and abiotic stresses (Carter 2011; Rodriguez et al. 2012). Some of these secondary endophytic metabolites with antimicrobial, antiparasitic, cytotoxic, anti-inflammatory, antitumor, antioxidant and neuroprotective activities (Aly et al. 2010; Gutierrez et al. 2012) are compounds possessing unique chemical structures and they have been studied for novel drug discovery (Mousa and Raizada 2013; Selim et al. 2012).

The resistance of bacteria to antibiotics has become a global concern and the search for new antibacterial agents is urgent and ongoing. The Infectious Diseases Society of America (IDSA) launched an initiative that supports

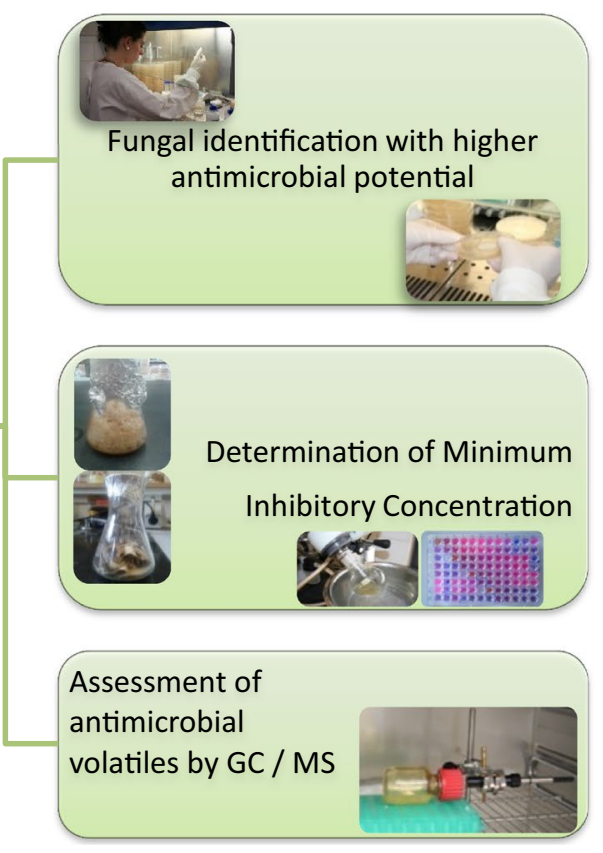

the development of 10 new classes of antibiotics by the year 2020 (IDSA 2010). Endophytic fungi are one of the potential sources of novel antimicrobial compounds, due to chemical diversity of their secondary metabolites. So far, studies reported a wide range of novel antimicrobial compounds produced by endophytic fungi belonging to diverse structural classes, including alkaloids, peptides, steroids, terpenoids, phenols, phenylpropanoids, aliphatic compounds, polyketides, quinones and flavonoids (Mousa and Raizada 2013; Yu et al. 2010), as well as volatile organic compounds (VOCs) such as esters, lipids, alcohols, acids, ketones, among others (Banerjee et al. 2010; Kudalkar et al. 2012). However, several studies have shown that the plant host, and ultimately its metabolism, influences the synthetic ability of endophytes (Strobel 2006; Ul-Hassan et al. 2012), which could prevent these microorganisms from being used in a commercial production of antimicrobial compounds (Kusari et al. 2014). Nevertheless, there still remain substantial questions about this issue. For instances, the genome sequencing and analysis of one paclitaxel-producing endophyte fungus revealed its ability to synthesize paclitaxel (Taxol $\left.{ }^{\mathrm{TM}}\right)$, an important anticancer drug, independently of the plant host (Yang et al. 2014). Similarly, several studies have shown that fungal Taxol is produced independently of the plant following several rounds of in vitro culturing (Guo et al. 2006; Soliman et al. 2011). The extent to which fungal endophytes produce natural metabolites apart of their host plant is not precise; and their elucidation is one of the key challenges to achieve the industrial production 
of desired bioactive metabolites from endophytes (Kusari et al. 2014).

Although a considerable body of research has investigated the antimicrobial metabolites synthesized by fungal endophytic isolated from various plants (Yu et al. 2010), screening of endophytic fungi from olive tree (Olea europaea L.) for antimicrobial activities has never been made. It has been estimated that only $5 \%$ of the world's fungi have so far been described (Maheshwari 2016), which suggested that a large number of new bioactive natural products from endophytic fungi still remains to be discover, in especially VOCs (Morath et al. 2012). Therefore, the current study was undertaken to screen the antimicrobial activity of three fungal endophytes isolated from olive tree leaves and evaluate the effect of the interaction between endophyte-host plant extracts on such activity. Also, the VOCs produced by the tested fungi were identified by gas chromatography/ mass spectrometry (GC/MS) and further correlated with the antimicrobial activity with an attempted to identify potential components responsible for the obtained antimicrobial activity. It is expected that the results obtained may contribute to the knowledge of new antimicrobial compounds produced by endophytic fungi.

\section{Materials and methods}

\section{Microorganisms and culture conditions}

The endophytic fungi tested, Penicillium commune Thom (MUM 15.02), Penicillium canescens Sopp (MUM 15.01) and Alternaria alternata (Fr.) Keissl. (MUM 16.02), were obtained from the fungal culture collection of the School of Agriculture, Polytechnic Institute of Bragança, Portugal. These isolates were originally obtained from leaves of $O$. europaea cv. Cobrançosa collected in the Trás-os-Montes region (northeast of Portugal) and were identified molecularly by sequencing the internal transcribed spacer (ITS) region of the nuclear ribosomal DNA (rDNA). Spores of these strains were stored in an aqueous glycerol solution $(30 \%, v / v)$ at $-80^{\circ} \mathrm{C}$. The fungal inoculum used in the assays was prepared from these frozen stocks by transferring spores onto potato dextrose agar (PDA) media. The fungus was grown in the dark at $25 \pm 1^{\circ} \mathrm{C}$, from 3 to 7 days, and conidial produced were used for the subsequent studies.

The antimicrobial activities were evaluated against three Gram-positive bacteria (Bacillus cereus ATCC 7064, Bacillus subtilis 48886 and Staphyloccocus aureus ATCC 6538), two Gram-negative bacteria (Escherichia coli CECT 423 and Pseudomonas aeruginosa ATCC 10145) and three yeasts (Candida albicans IGC 3436T, Candida glabrata IGC 2418T and Candida parapsilosis 28B). All the microorganisms were obtained from the Biology
Department-University of Minho (Braga, Portugal). Yeast strains were maintained at $4{ }^{\circ} \mathrm{C}$ in YEPDA medium [1\% $(\mathrm{w} / \mathrm{v})$ yeast extract, $2 \%(\mathrm{w} / \mathrm{v})$ peptone, $2 \%(\mathrm{w} / \mathrm{v})$ glucose and $2 \%(\mathrm{w} / \mathrm{v})$ agar], and sub-cultured periodically. Yeasts were grown aerobically at $28{ }^{\circ} \mathrm{C}$. Bacterial stocks cultures were maintained at $4{ }^{\circ} \mathrm{C}$ on LB agar [tryptone $1 \%(\mathrm{w} / \mathrm{v})$, yeast extract $0.5 \%(\mathrm{w} / \mathrm{v}), \mathrm{NaCl} 1 \%(\mathrm{w} / \mathrm{v})$ and agar $2 \%$ (w/v)], being sub-cultured periodically at $37^{\circ} \mathrm{C}$.

\section{Screening of fungal endophytes for antimicrobial activity}

The screening of fungal endophytes for antimicrobial activity was performed in two steps. In the first step, endophytic fungi were screen for their antimicrobial activity on PDA medium with or without aqueous extract of olive tree leaves, and results were compared in order to investigate the effect of the interaction between endophytic fungi and plant compounds in the antimicrobial activity. In this primary screen was used the bioassay method developed by Pereira et al. (2013). In the second step, was calculated the minimum inhibitory concentration (MIC). This secondary screen was done by the micro-dilution method (Rios et al. 1988) with several solvents extracts of the cultured broth and mycelium of the fungus, to determine if the antimicrobial compounds were in the liquid culture secreted from the mycelium or inside the mycelium.

\section{Preparation of plant extracts}

Plant extract was prepared with fresh leaves collected from healthy olive trees from cv. Cobrançosa, located in Mirandela (northeast of Portugal). The collected leaves were transported to the laboratory in an icebox, and immediately upon arrival, they were rinsed in distilled water, ground to a fine powder in liquid nitrogen and stored at $-80^{\circ} \mathrm{C}$. The plant extract was prepared by stirring $2 \mathrm{~g}$ of fresh ground leaves in $100 \mathrm{~mL}$ distilled water, at $150 \mathrm{rpm}$ and $25^{\circ} \mathrm{C}$, for $30 \mathrm{~min}$. After filtration through a Whatman filter paper No. 4 , the aqueous leaf extract was used to prepare PDA medium.

\section{Bioassay method}

The antimicrobial activity assay was performed in Petri dishes $(9 \mathrm{~cm}$ diameter) containing PDA medium prepared with distilled water or prepared with aqueous extract of host plant leaves $(2 \%, \mathrm{w} / \mathrm{v})$. Both culture media (PDA with and without host plant extract) were inoculated by transferring $10 \mu \mathrm{L}$ of a spore suspension $\left(1 \times 10^{6}\right.$ spore $\left./ \mathrm{mL}\right)$ of each $P$. canescens, $P$. commune and A. alternata fungus to the centre of a Petri dish ( $9 \mathrm{~cm}$ diameter). Spore suspensions were obtained by flooding fungal cultures growing 
on PDA medium with $2 \mathrm{~mL}$ of sterile aqueous solution of $0.02 \%(\mathrm{v} / \mathrm{v})$ Tween 80 . After incubation at $25 \pm 1{ }^{\circ} \mathrm{C}$, in the dark, for 3 ( $P$. commune), 6 (A. alternata) and 8 days $(P$. canescens), the inoculated plates were overlaid with the sensitive indicator strain. This was obtained by scraping biomass of yeast and bacteria species from a $24 \mathrm{~h}$ YPDA or LB culture plate, respectively, that was further suspended in $1 \mathrm{~mL}$ of $\mathrm{NaCl} 0.85 \%(\mathrm{w} / \mathrm{v})$. The titre of yeast/bacteria suspension was calculated using a Neübauer haemacytometer, and their concentration was adjusted to $10^{8} \mathrm{CFU}$ (colony forming units) $/ \mathrm{mL}$. This suspension was mixed with melted-agar $0.8 \%(\mathrm{w} / \mathrm{v})$ in order to obtain $10^{6} \mathrm{CFU} / \mathrm{mL}$. A volume of $5 \mathrm{~mL}$ of the mixture was then seeded as a lawn onto the surface of the plates previously inoculated with endophytic fungi. The plates were incubated at $25^{\circ} \mathrm{C}$ for $48 \mathrm{~h}$ and $37^{\circ} \mathrm{C}$ for $24 \mathrm{~h}$ for yeasts and bacteria, respectively. Standard discs of chloramphenicol $(30 \mu \mathrm{g} / \mathrm{mL})$ and fluconazole $(25 \mu \mathrm{g} / \mathrm{mL})$ (Oxoid Ltd.) were used as positive controls for antibacterial and antifungal activity, respectively. Antimicrobial activity was observed when the fungal inoculum was surrounded by a clear zone of growth inhibition and evaluated by determining both the diameter of the fungal colony and the diameter of the inhibition halo. Values were used to calculate the areas occupied by the fungus and by the inhibition halo formed by its antimicrobial activity, which were further subtracted to obtain the inhibition halo area. Data are presented as the mean of three independent experiments, with three replicas each. The corresponding standard error values are displayed.

\section{Micro-dilution method}

The endophyte that showed the highest antimicrobial activity, especially towards yeasts, in the first screen (i.e. A. alternata) was cultured in potato dextrose broth (PDB) medium, and MIC values were determined for ethyl acetate, methanol and methanol:water $(1: 1, \mathrm{v} / \mathrm{v})$ extracts of both mycelium and cultured broth. MIC values were also evaluated for PDB medium extracts that was used as control. Submerged cultures were grown in $250 \mathrm{~mL}$ Erlenmeyer flasks with $150 \mathrm{~mL}$ of $\mathrm{PDB}$ each, at $25^{\circ} \mathrm{C}$ for 10 days, in the dark and without agitation. After incubation, the culture broth and fungal biomass were separated by filtration through two layers of Miracloth (22-25 $\mu \mathrm{m}$ pore size) and were further freeze-dried. The freeze-dried mycelium/culture broth/PDB ( $3 \mathrm{~g}$ ) were extracted by stirring with $100 \mathrm{~mL}$ of each solvent [ethyl acetate, methanol and methanol:water $(1: 1, \mathrm{v} / \mathrm{v})]$, at room temperature, at $150 \mathrm{rpm}$ for $1 \mathrm{~h}$, and filtered through Whatman filter paper No. 4. Extraction was additionally repeated two times, and the filtrates obtained for each solvent were combined. The solvent was removed by evaporation under vacuum on reduced pressure at $40-45^{\circ} \mathrm{C}$ using a rotary evaporator.
After evaporation, the remaining water was removed by lyophilisation. The extracts obtained were weighted and dissolved in 10\% (v/v) dimethyl sulfoxide (DMSO) at concentrations ranging from 25 to $350 \mathrm{mg} / \mathrm{mL}$, depending on the extraction yield, and further used to determine MIC values. MIC values of the several extracts were determined by a broth micro-dilution assay in PDB medium at $25^{\circ} \mathrm{C}$. Each well containing $100 \mu \mathrm{L}$ of twofold serial dilutions of the extract (concentration are indicated in Table S1) was inoculated with $100 \mu \mathrm{L}$ of yeast or bacteria inoculum at $10^{6} \mathrm{CFU} / \mathrm{mL}$. Chloramphenicol $(30 \mu \mathrm{g} / \mathrm{mL})$ and fluconazole $(25 \mu \mathrm{g} / \mathrm{mL})$ were used as positive controls for antibacterial and antifungal activity, respectively; and a control with $10 \%$ (v/v) DMSO and yeast/bacteria inoculum was also included. All experiments were performed in triplicate and repeated twice. After incubation for $24 \mathrm{~h}$ at $25^{\circ} \mathrm{C}$, $10 \mu \mathrm{L}$ of $0.15 \mathrm{mg} / \mathrm{mL}$ resazurin (Sigma) was added to each well, and further incubated for $2 \mathrm{~h}$ for the observation of colour change. The wells with a color change from purple to pink or colorless are indicative of positive bacterial/yeast growth while wells remaining purple represent no microbial growth. The MIC was recorded as the lowest concentration of extract that inhibited the growth of bacteria/yeast. The results were expressed in $\mathrm{mg}$ per $\mathrm{mL}$ and presented as the mean of three independent experiments.

\section{Analysis of volatile compounds}

The analysis of volatile composition was studied in the species that showed higher and lower antimicrobial activity, respectively A. alternata and $P$. canescens (see "Results" and "Discussion" sections). The in vitro assays were performed in PDA medium.

\section{In vitro fungal cultures}

The two fungi species $(10 \mu \mathrm{L}$ of a spore suspension at $1 \times 10^{6}$ spore $/ \mathrm{mL}$ ) were grown in $50 \mathrm{~mL}$ flasks (Duran Gaines Synth, Bioblock) (6 days for A. alternata and 8 days for $P$. canescens at $25^{\circ} \mathrm{C}$ and in dark), containing $10 \mathrm{~mL}$ of PDA medium, sealed with a polypropylene cap with PTFE/ silicon septum (Duran). Four replicates per fungal species were performed; and flasks containing exclusively PDA medium were also included as control.

\section{Extraction of volatile compounds}

The extraction of volatile compounds from in vitro cultures of $P$. canescens and A. alternata was performed by headspace solid-phase microextraction (HS-SPME). In the $50 \mathrm{~mL}$ vials containing the fungus a SPME fiber coated with divinylbenzene/carbonex/polydimethylsiloxane (DVB/ CAR/PDMS 50/30 $\mu \mathrm{m}$ ) (Supelco, Bellefonte, USA) was 
used. The entire procedure was done in a horizontal position in a gas emission system (isolation) at $40{ }^{\circ} \mathrm{C}$. The vials with the respective fungus were placed $10 \mathrm{~min}$ in an oven at $40^{\circ} \mathrm{C}$ for an incisive release of the volatile compounds. After this period, the SPME fiber was exposed during $60 \mathrm{~min}$ for the compounds adsorption in the headspace. The fiber was then inserted in the injection port of the chromatography system. The HS-SPME procedure was made in quadruplicate with a control sample (only PDA medium).

\section{Gas chromatography-mass spectrometry (GC/MS) analysis}

The retained compounds were eluted from the fiber by thermal adsorption for $1 \mathrm{~min}$. For cleaning and conditioning of further analyzes the fiber was maintained during $10 \mathrm{~min}$ at $220^{\circ} \mathrm{C}$ in the injector port of the chromatography system. The gas chromatographer used was a Shimadzu GC-2010 Plus equipped with a mass spectrometer Shimadzu GC/MS-QP2010 SE detector. A TRB-5MS $(30 \mathrm{~m} \times 0.25 \mathrm{~mm} \times 0.25 \mu \mathrm{m})$ column (Teknokroma, Spain) was used. The injector was set at $220^{\circ} \mathrm{C}$ and the manual injections were made in splitless mode. The mobile phase consisted in helium (Praxair, Portugal) at a linear velocity of $30 \mathrm{~cm} / \mathrm{s}$ and a total flow of $24.4 \mathrm{~mL} / \mathrm{min}$. The oven temperatures were the following: $40^{\circ} \mathrm{C} / 1 \mathrm{~min} ; 2^{\circ} \mathrm{C} / \mathrm{min}$ until $220^{\circ} \mathrm{C} ; 220^{\circ} \mathrm{C}$ during $30 \mathrm{~min}$. The ionization source was maintained at $250^{\circ} \mathrm{C}$ with an ionization energy of $70 \mathrm{eV}$, and with an ionization current of $0.1 \mathrm{kV}$. All mass spectra were acquired by electron ionization. The ionization was left off during the first $3 \mathrm{~min}$. The MS spectra fragments were compared with those obtained from a database (NIST 11). For quantification purposes, each sample was injected in quadruplicate, and the areas of the chromatographic peaks were determined integrating the re-constructed chromatogram from the full scan chromatogram using for each compound the ion base ( $\mathrm{m} / \mathrm{z}$ intensity $100 \%)$.

\section{Data analysis}

Principal components analysis (PCA) was performed with data of volatile composition of $P$. canescens and A. alternata and of their antimicrobial activity (diameter of the inhibition halo) against several microorganisms (6 variables corresponding to volatile components, and 8 variables corresponding to inhibition halo values; with a total of 14 variables), with an attempt to describe the relationship between these two parameters (volatile compounds vs. antimicrobial activity). Significant differences among samples were verified using one-way ANOVA with a Tukey post-hoc test at $p$-value $<0.05$. All these analyses were conducted with SPSS software, version 21.0 (IBM Corporation, New York, USA).

\section{Results}

\section{Antimicrobial activity of endophytes and effect of host plant extract}

The antimicrobial activity of the endophytes $P$. commune, A. alternata, and $P$. canescens isolated from olive tree was tested against several pathogenic bacteria and yeast in the presence or absence of the host plant extracts (Fig. 1). In medium without olive leaf extract all bacteria under analysis, with exception of $E$. coli, were significantly inhibited by the three endophytes when compared to the control (Fig. 1b, c). Both P. commune and P. canescens were the most active against bacteria inhibiting up to 2.7 -fold when compared to the control. The first specie was the most effective in inhibiting B. cereus, $P$. aeruginosa and $C$. parapsilosis (up to 3.1-, 3.5- and 11.2-fold, respectively when compared to the control), whereas $P$. canescens was found to be the most efficient in inhibiting $E$. coli, $S$. aureus and $B$. subtilis (up to 2.5-, 2.7- and 2.9-fold, respectively when compared to the control). Although A. alternata inhibited significantly the bacteria to grow (B. cereus up to 2.4-fold, B. subtilis up to 2.2-fold, $S$. aureus up to 2.4-fold and $P$. aeruginosa up to 3.0-fold, when compared to chloramphenicol), it showed higher activity against yeasts (Fig. 1a). When compared with the control fluconazole, A. alternata reduced significantly the growth of $C$. albicans (up to 8.2fold), C. glabrata (up to 7.1-fold) and C. parapsilosis (up to 9.0 -fold). The endophyte A. alternata also displayed the widest antimicrobial spectrum (inhibited significantly 7 out of 8 microorganisms analyzed) and was the only endophyte tested with capacity to inhibit significantly $C$. albicans more than the control.

The incorporation of host plant extract into the culture medium showed to play an important role in the antimicrobial activity, depending on the endophytic species. When considering the results obtained for all the pathogens analyzed, only the antimicrobial activity of $P$. commune grown on medium with host extracts exhibited significant changes compared to medium without olive leaf extract $\left(\mathrm{F}_{1,190}=21.140, p<0.001\right.$; Table 1$)$. The antimicrobial activity displayed in the assay with this specie increased significantly (in average 1.4-fold), especially against yeasts (in average 2.4-fold; $\mathrm{F}_{1,70}=34.083, p<0.001$ ) and Gram-negative bacteria (in average 1.2-fold; $\mathrm{F}_{1,46}=4.954$, $p<0.05$ ), upon addition of host extract to the culture medium (Fig. 1; Table 1). The antimicrobial activity in the assays with both $P$. canescens and A. alternata seems to be less influenced by host extract than $P$. commune, since no significant differences were found between the presence and absence of host extract on culture medium when considering the results obtained for all the pathogens analyzed (Table 1). Despite this, host extract showed to influenced 
Without olive leaf extract
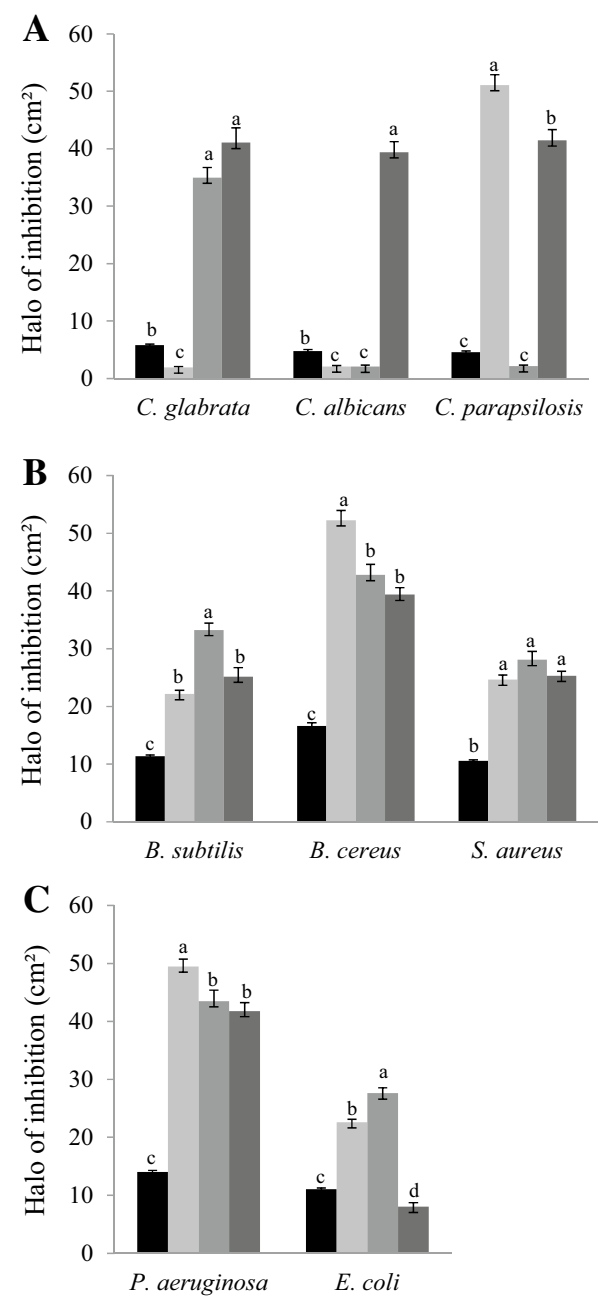

With olive leaf extract
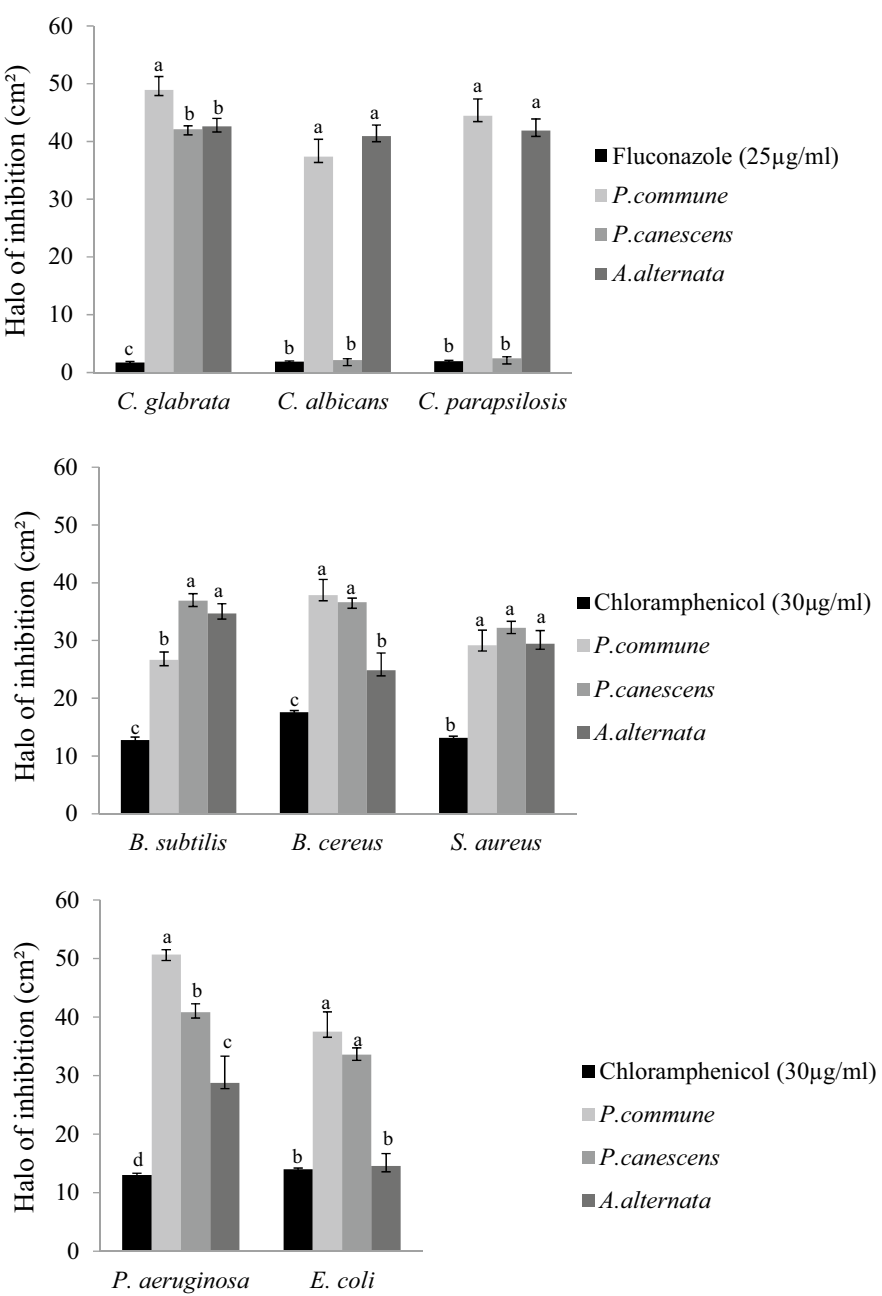

Fig. 1 Antimicrobial activity (halo of inhibition, in area) against yeasts (a), Gram-positive bacteria (b) and Gram-negative bacteria (c) displayed by the endophytic fungi Penicillium commune, Penicillium canescens and Alternaria alternata, cultured in medium without and with olive leaf extract. Each value is expressed as mean \pm standard error of three independent experiments performed in triplicate. Bars with different letters indicate values with significant differences at $p<0.05$, within each microorganism significantly the antimicrobial activity in the assays with $P$. canescens against five microorganisms (one yeast, three Gram-positive bacteria and one Gram-negative bacteria) and of A. alternata against four bacteria (two Gram-positive and two Gram-negative), out of the eight microorganisms analyzed.

The incorporation of plant host extract into de culture medium may induce or repress the antimicrobial activity (Fig. 1; Table 1). Plant host extract significantly enhanced the antimicrobial activity of $P$. commune and $P$. canescens against $C$. glabrata (25.4- and 1.2-fold, respectively), C. albicans (17.8-fold for the first specie), B. subtilis (1.2- and 1.1-fold, respectively), S. aureus (1.1-fold for the former specie) and E. coli (1.7- and 1.2-fold, respectively), but decrease against $B$. cereus (1.4- and 1.2-fold, respectively). Although with less effect compared to Penicillium strains, the host extract similarly shown to significantly increase the antimicrobial activity of A. alternata against B. subtilis (1.4-fold) and E. coli (1.8-fold), and to reduce against B. cereus (1.6-fold) and $P$. aeruginosa (1.5-fold). Curiously, was also noticed that in the presence of host extract, the antimicrobial activity displayed by all the endophytes was significantly enhanced against $B$. subtilis (in average 1.2-fold) and $E$. coli (in average 1.6-fold), but reduced against $B$. cereus (in average 1.4-fold). 
Table 1 Effect of olive tree leaf on the antimicrobial activity displayed by endophytic fungi

\begin{tabular}{lllll}
\hline Microorganisms & & \multicolumn{2}{l}{ Endophytic fungi } \\
\cline { 3 - 5 } & & P. commune & P. canescens & A. alternata \\
\hline Yeasts & & & & \\
C. glabrata & $\mathrm{F}(1,22)$ & $421.255^{* * *}$ & $14.649^{* * *}$ & $0.296^{\mathrm{ns}}$ \\
C. albicans & $\mathrm{F}(1,22)$ & $139.074^{* * *}$ & $0.100^{\mathrm{ns}}$ & $0.335^{\mathrm{ns}}$ \\
C. parapsilosis & $\mathrm{F}(1,22)$ & $3.776^{\mathrm{ns}}$ & $1.106^{\mathrm{ns}}$ & $0.025^{\mathrm{ns}}$ \\
Total & $\mathrm{F}(1,70)$ & $34.083^{* * *}$ & $0.366^{\mathrm{ns}}$ & $0.335^{\mathrm{ns}}$ \\
Gram+ & & & & \\
B. subtilis & $\mathrm{F}(1,22)$ & $8.684^{* *}$ & $4.723^{*}$ & $17.850^{* * *}$ \\
B. cereus & $\mathrm{F}(1,22)$ & $20.055^{* * *}$ & $9.605^{* *}$ & $20.490^{* * *}$ \\
S. aureus & $\mathrm{F}(1,22)$ & $2.740^{\mathrm{ns}}$ & $4.808^{*}$ & $3.034^{\mathrm{ns}}$ \\
Total & $\mathrm{F}(1,70)$ & $0.396^{\mathrm{ns}}$ & $0.127^{\mathrm{ns}}$ & $0.016^{\mathrm{ns}}$ \\
Gram- & & & & \\
P. aeruginosa & $\mathrm{F}(1,22)$ & $0.573^{\mathrm{ns}}$ & $1.266^{\mathrm{ns}}$ & $7.410^{*}$ \\
E. coli & $\mathrm{F}(1,22)$ & $19.304^{* * *}$ & $15.481^{* * *}$ & $8.741^{* *}$ \\
Total & $\mathrm{F}(1,46)$ & $4.954^{*}$ & $0.519^{\mathrm{ns}}$ & $4.956^{*}$ \\
Total & $\mathrm{F}(1,190)$ & $21.140^{* * *}$ & $0.461^{\mathrm{ns}}$ & $2.417^{\mathrm{ns}}$ \\
\hline
\end{tabular}

$F$ values and probability levels of the effect of olive tree leaf on the antimicrobial activity against Gram-positive bacteria, Gram-negative bacteria and yeasts displayed by the endophytic fungi $P$. commune, $P$. canescens and A. alternata

Probability levels: n.s. no significant, $* p<0.05, \quad * * p<0.01$, $* * * p<0.001$

\section{Determination of minimum inhibitory concentration (MIC)}

During the initial screening, only A. alternata exhibited a potent broad spectrum activity against yeasts, including C. albicans, independently of the host plant extract. This feature makes this specie an ideal candidate to be explored as a microbial factory for industrial production of desirable antimicrobial compounds. Therefore, we have selected this specie to determine the MICs values of both mycelium and cultured broth extracts, by using a broth micro-dilution method. The results depicted in Table 2 showed that microorganism sensitivity differed with the nature of the organic solvent used to extract active fractions. Ethyl acetate extract exhibited a stronger broad spectrum activity when compared to methanol and methanol:water $(1: 1, \mathrm{v} / \mathrm{v})$. Ethyl acetate extracts inhibited all the tested strains at MIC ranging from 0.095 to $25 \mathrm{mg} /$ $\mathrm{mL}$, being more active against yeasts (MIC $\leq 0.095 \mathrm{mg}$ / $\mathrm{mL}$ ), followed by bacteria (MIC $\leq 3.125 \mathrm{mg} / \mathrm{mL}$ for Gramnegative and MIC $\leq 25.000 \mathrm{mg} / \mathrm{mL}$ for Gram-positive). By contrast, both methanol and methanol:water $(1: 1, \mathrm{v} / \mathrm{v})$ only inhibited the Gram-positive bacteria B. cereus at the MIC range of $0.351-0.683$ and $0.080-0.930 \mathrm{mg} / \mathrm{mL}$, respectively (Table 2). These findings suggested that most active fraction of endophytic fungi was extracted in ethyl acetate rather than other organic solvents. It was also noticed a lack of growth inhibition of all microorganisms by the PDB medium extract (control) prepared from the several organic solvents (data not shown).

The results also indicate that, in general, the antimicrobial metabolites produced by A. alternata were inside the mycelium or secreted into the culture medium. In fact, ethyl acetate extract of the mycelium was found to be as effective as the culture broth of $A$. alternata in inhibiting $S$. aureus, B. cereus, C. glabrata, C. parapsilosis and C. albicans (Table 2). For some microorganisms, such as B. subtilis, E. coli and B. cereus, the extracts prepared from mycelium presented a higher inhibition level when compared to extracts from culture broth. The Gram-negative bacteria $P$. aeruginosa was an exception, being only inhibited by ethyl acetate extract prepared from culture broth.

\section{Volatile profile}

We have analyzed the VOCs produced by A. alternata by GC/MS in an attempt to identify the compounds potentially

Table 2 MIC values ${ }^{\mathrm{a}}$ of the extracts prepared from mycelium and cultured broth of Alternaria alternata

\begin{tabular}{|c|c|c|c|c|c|c|c|c|}
\hline & B. subtilis & S. aureus & B. cereus & P. aeruginosa & E. coli & C. glabrata & C. parapsilosis & C. albicans \\
\hline \multicolumn{9}{|l|}{ Ethyl acetate } \\
\hline Culture broth & 25.000 & 6.250 & 0.095 & 3.125 & 3.125 & 0.095 & 0.095 & 0.095 \\
\hline Mycelium & 0.095 & 6.250 & 0.095 & - & 0.095 & 0.095 & 0.095 & 0.095 \\
\hline \multicolumn{9}{|c|}{ Methanol:water $(1: 1, \mathrm{v} / \mathrm{v})$} \\
\hline Culture broth & - & - & 0.930 & - & - & - & - & - \\
\hline Mycelium & - & - & 0.080 & - & - & - & - & - \\
\hline \multicolumn{9}{|l|}{ Methanol } \\
\hline Culture broth & - & - & 0.683 & - & - & - & - & - \\
\hline Mycelium & - & - & 0.351 & - & - & - & - & - \\
\hline
\end{tabular}

- No inhibition

${ }^{\mathrm{a}} \mathrm{MIC}$ values $(\mathrm{mg} / \mathrm{mL})$ of the extracts prepared from mycelium and cultured broth of $A$. alternata cultures 
responsible for the antimicrobial activity displayed by this specie. The volatile profile of the endophytic specie that displayed the lowest activity against yeasts (i.e. P. canescens) was also evaluated and used as a negative control. The chromatographic volatile profile (Table 3; Fig. 2) revealed that six compounds were found among A. alternata and $P$. canescens. The VOCs formally and tentatively identified belong to different chemical classes: three alcohols (3-methyl-1-butanol, 1-octen-3-ol, and phenylethyl alcohol), one ester (3-methyl-1-butanol acetate), and two sesquiterpenes ( $\beta$-cedrene and thujopsene). The results revealed differences among endophytes, both in compounds identified as well as in their amounts. The volatile fraction of A. alternata was composed by five compounds and $P$. canescens by three compounds (Table 3 ). The most abundant volatile identified in both endophytes was 3-methyl1-butanol (63.4 and $66.6 \%$ of the total volatile fraction, respectively) followed by phenylethyl alcohol $(22.4$ and $20.2 \%$, respectively). 3-Methyl-1-butanol acetate (7.2\%), was the third most abundant compound found in A. alternata, followed by thujopsene (6.2\%) and $\beta$-cedrene (0.7\%); whereas in P. canescens 1-octen-3-ol (13.1\%) was the third most abundant compound identified, being this mushroomlike alcohol exclusively found in this fungus.

A principal component analysis was performed with volatile profile obtained from these two fungi and their antimicrobial activity (halo inhibition) against bacteria and yeasts, to describe the relationship between the two parameters (Fig. 3). An interesting result is the clustering of $C$. albicans and C. parapsilosis together with the volatile compounds 3-methyl-1-butanol, phenylethyl alcohol, 3-methyl1-butanol acetate, $\beta$-cedrene, and thujopsene, suggesting their involvement in the inhibition displayed by $A$. alternata against those yeasts. The cluster of 1-octen-3-ol together with $E$. coli, B. subtilis and $P$. aeruginosa, suggested the participation of this compound in the observed inhibitory effect displayed by Penicillium canescens against those bacteria.

\section{Discussion}

Drug resistance among microbes is well recognized as a major threat to human health, and the development of new antimicrobial agents is pointed out as one of the effective solutions to address this problem (Laximanarayan et al. 2013). The endophytes tested in the present study (i.e. $A$. alternata, $P$. canescens and $P$. commune) could be of considerable interest to the development of new drugs against an array of human pathogenic microorganisms. Both $P$. canescens and $P$. commune were found to be the most potent against bacteria, whereas A. alternata showed higher activity against yeasts. Several species belonging to the genus Penicillium have already been reported as being prolific producers of bioactive secondary metabolites (Nicoletti et al. 2014), including of antimicrobial compounds (Yu et al. 2010). However, to our knowledge, the antimicrobial potential of the endophytes $P$. commune (Moghaddam et al.

Table 3 Volatile profile of the endophytic fungi Penicillium canescens and Alternaria alternata

\begin{tabular}{|c|c|c|c|c|c|c|c|c|c|}
\hline & \multirow[t]{2}{*}{ Compound } & \multirow[t]{2}{*}{$\mathrm{RT}^{\mathrm{A}}$} & \multirow[t]{2}{*}{$\mathrm{LRI}^{\mathrm{B}}$} & \multirow{2}{*}{$\begin{array}{l}\text { Characteristic ions } \\
(\mathrm{m} / \mathrm{z})\end{array}$} & \multirow[t]{2}{*}{$\mathrm{QI}(\mathrm{m} / \mathrm{z} 100 \%)^{\mathrm{C}}$} & \multirow{2}{*}{$\begin{array}{l}\text { Molecu- } \\
\text { lar } \\
\text { weight }\end{array}$} & \multirow{2}{*}{$\begin{array}{l}\text { Molecular } \\
\text { formula }\end{array}$} & \multicolumn{2}{|c|}{ Area/ $1000 \pm S^{D}$} \\
\hline & & & & & & & & P. canescens & A. alternata \\
\hline 1 & $\begin{array}{l}\text { 3-Methyl-1-bu- } \\
\text { tanol }\end{array}$ & 4.7 & 697 & $42 / 55 / 70$ & 55 & 88 & $\mathrm{C}_{5} \mathrm{H}_{12} \mathrm{O}$ & $10,115 \pm 3151^{\mathrm{a}}$ & $25,585 \pm 11,517^{\mathrm{b}}$ \\
\hline 2 & $\begin{array}{l}\text { 3-Methyl-1-bu- } \\
\text { tanol acetate }\end{array}$ & 9.8 & 820 & $43 / 55 / 70$ & 43 & 130 & $\mathrm{C}_{7} \mathrm{H}_{14} \mathrm{O}_{2}$ & n.d. & $2697 \pm 467$ \\
\hline 3 & 1-Octen-3-ol & 15.48 & 969 & 57 & 57 & 128 & $\mathrm{C}_{8} \mathrm{H}_{16} \mathrm{O}$ & $1901 \pm 433$ & n.d. \\
\hline 4 & $\begin{array}{l}\text { Phenylethyl } \\
\text { alcohol }\end{array}$ & 24.08 & 1136 & $91 / 92 / 122$ & 91 & 122 & $\mathrm{C}_{8} \mathrm{H}_{10} \mathrm{O}$ & $3080 \pm 986^{\mathrm{a}}$ & $9065 \pm 4189^{b}$ \\
\hline 5 & $\beta$-Cedrene & 44.70 & 1398 & $41 / 69 / 93 / 120 / 161 / 204$ & 161 & 204 & $\mathrm{C}_{15} \mathrm{H}_{24}$ & n.d. & $346 \pm 338$ \\
\hline 6 & Thujopsene & 45.40 & 1416 & 41/93/105/119/133 & 119 & 204 & $\mathrm{C}_{15} \mathrm{H}_{24}$ & n.d. & $2313 \pm 1653$ \\
\hline
\end{tabular}

Volatile profile of the endophytic fungi $P$. canescens and A. alternata growing under in vitro conditions, expressed in chromatographic normalized peak area (mean $\pm \mathrm{SD}, \mathrm{n}=4$ ). Compounds found in the uninoculated PDA medium (control) are not included in this table and were used to subtract any possible compounds from the medium. Different superscript lower case letters denote statistically significant differences $(p<0.05)$ between fungal species

n.d. not detected

${ }^{\text {A }}$ Retention time

${ }^{\mathrm{B}}$ Linear Retention Index reported in literature (NIST 11)

${ }^{\mathrm{C}}$ Quantification ion

${ }^{\mathrm{D}}$ Area expressed as arbitrary units (mean of four replicates \pm standard deviation) 

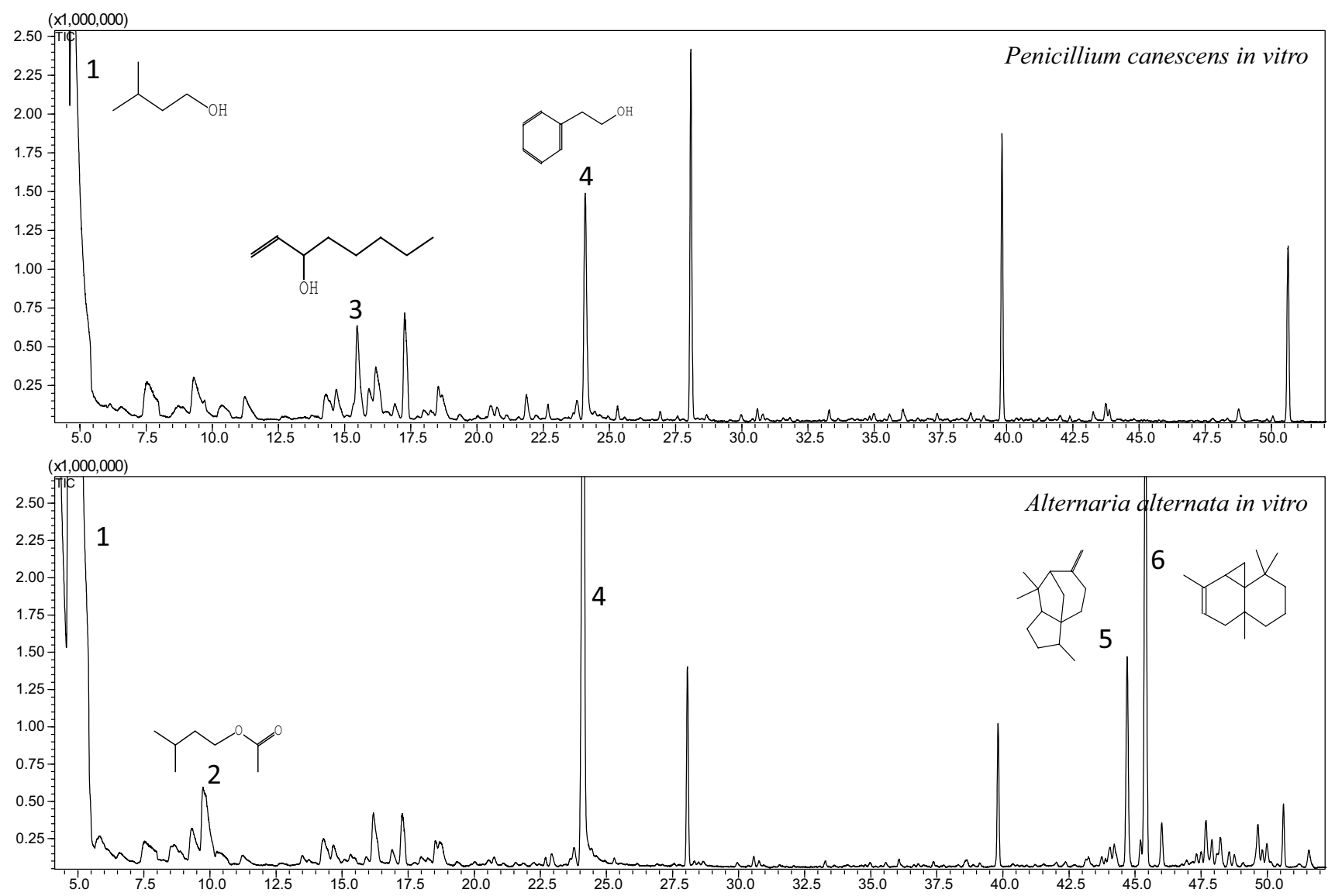

Fig. 2 Full scan chromatographic profile obtained from the endophytic fungi Penicillium canescens and Alternaria alternata growing in vitro by HS-SPME using DVB/CAR/PDMS fiber (identification numbers correspond to those compounds reported in Table 3)

2013) and P. canescens (Bertinetti et al. 2009; Nicoletti et al. 2014) was only explored against phytopathogenic fungi. Studies focusing antimicrobial activity of $P$. commune against human pathogens are mainly performed with marine sediment-derived strains (Gao et al. 2011). Thus, the present study provides, for the first time, evidences of the antimicrobial potentialities of both endophytes $P$. commune and $P$. canescens against human pathogens, and exhorts their potential use in the treatment of infections caused mostly by bacteria. This is of particular importance for E. coli and P. aeruginosa, due to increased levels of resistance of these bacteria to multiple classes of antibiotics. Actually, the multidrug resistance is an acute problem and treatment options are particularly limited (Högberg et al. 2010).

The endophyte A. alternata showed strong activity against all the Candida tested, including of $C$. albicans, which make this fungus a candidate for testing in the treatment of this kind of infection. This endophyte may also have potential utility as antibacterial agent due to its capacity to inhibit significantly the bacteria $B$. cereus, B. subtilis, S. aureus and P. aeruginosa. Extracts of A. alternata inhabiting coffee plants (Fernandes et al. 2009), silver trumpet tree (Sadananda et al. 2011) and sweet wormwood (Qadri et al. 2013) have been previously found to displayed antimicrobial activity against an array of bacteria and yeasts, including C. albicans (Qadri et al. 2013). The various organic extracts from both A. alternata mycelium and cultured broth were also found to displayed different antimicrobial effects. The highest antimicrobial activity found in the ethyl acetate extract compared to the other solvents was previously observed for several endophytic fungi species (Musavi and Balakrishnan 2014; Verma et al. 2008). In a general way, and according to the MIC values obtained, ethyl acetate should be selected as the best extraction solvent to obtain optimized antimicrobial agents from endophytic fungus. We also found that ethyl acetate extracts of A. alternata mycelium and culture broth were more active against yeast (MIC $\leq 0.095 \mathrm{mg} / \mathrm{mL}$ ) than against bacteria (MIC ranging from 0.095 to $25 \mathrm{mg} / \mathrm{mL}$ ). Most of our MIC values were in range or slightly lower than those reported by Fernandes et al. (2009) for crude extract of $A$. alternata against $S$. aureus, $E$. coli and $C$. albicans (MIC of range $0.05-0.1,0.4-0.8$ and $>0.8 \mathrm{mg} / \mathrm{mL}$, respectively) 


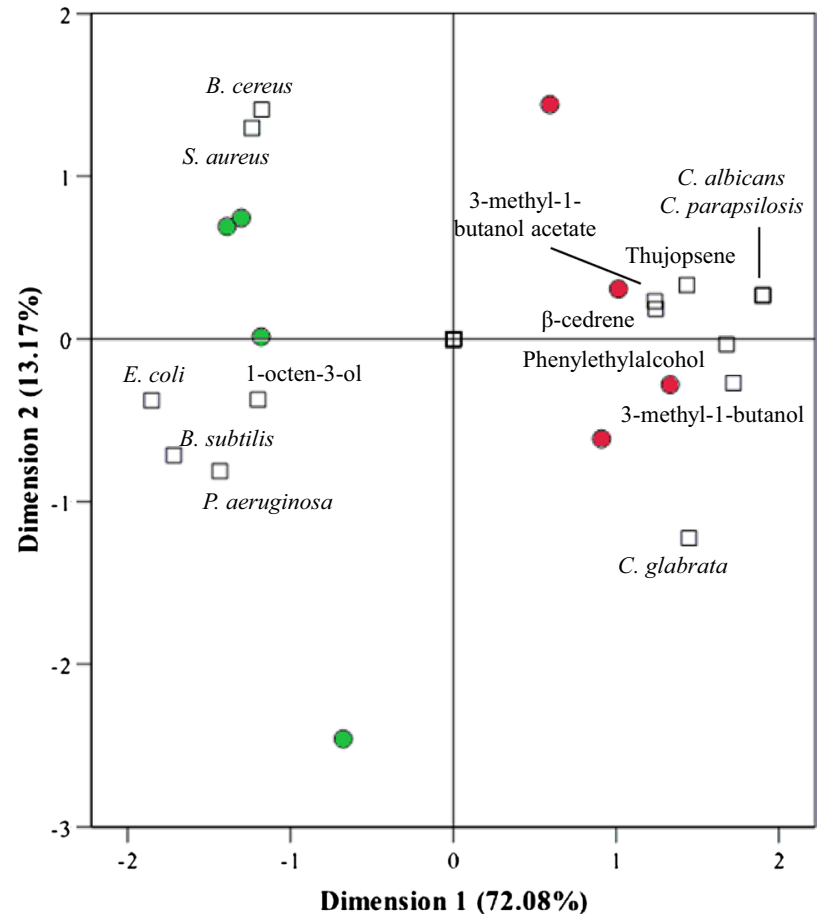

Fig. 3 Principal component analysis obtained using the volatile composition of Alternaria alternata (red circle) and Penicillium canescens (green circle) growing in vitro and antimicrobial activity displayed by these fungi against bacteria and yeasts, evaluated as halo inhibition $\left(\right.$ in $\mathrm{cm}^{2}$ ). The PCA factors explain $85.25 \%$ of the total variance. (Color figure online)

and by Ramesha et al. (2013) for ethyl acetate extract of A. alternata against $S$. aureus, B. cereus, E. coli and $P$. aeruginosa (MIC $=0.02 \mathrm{mg} / \mathrm{mL}$ ). Some of the antimicrobial compounds produce by fungi belonging to the genus Alternaria have been already identified. For instance, altenusin extracted from Alternaria spp. mycelium, exhibit antimicrobial activity against several multidrug-resistant bacterial and fungal strains (Kjer et al. 2009). Similarly, altersetin isolated from the culture broth of Alternaria spp. have showed potent activity against Gram-positive bacteria (Hellwig et al. 2002).

The role of host plant extract on the biological activity of fungal endophytes is not precisely known. Such knowledge would favor the industrial utilization and commercial production of biologically useful compounds by endophytic fungi. Our results indicate that host plant extract had influence in the antimicrobial activity displayed by the fungal endophytes studied, in special of the $P$. commune. The presence of host plant in the medium was showed to stimulate or repress the antimicrobial activity, according to the microorganisms assayed. For instances, in the presence of host plant extract, the antimicrobial activity of $P$. commune increased significantly against some Candida spp. but decreased against some bacteria. Among the endophytes tested, A. alternata seems to be less influenced by host extract. These results suggest that each of the three endophytic fungi tested may release different metabolites in the presence of the microorganisms and synergic or antagonistic effects may be seen in the presence of leaf aqueous extract from cv. Cobrançosa. Leaves of this cultivar are described to be rich in antimicrobial components like phenolic compounds, namely secoiridois (mainly oleuropein) (Pereira et al. 2007) and flavonoids (mainly luteolin 4-O-glucoside) (Meirinhos et al. 2005). However, the common feature of all endophytes in the antimicrobial activity displayed against B. subtilis, E. coli and B. cereus upon addition of host extract in culture media suggested that the nature of some of the antimicrobial metabolites could also be common among the endophytes tested. In the future, fractionation based bioassays using olive leaf extracts may be used to identify candidate diffusible metabolites that affect endophytic antimicrobial biosynthesis. Previous studies have similarly found that host plant-derived compounds are required for the synthesis of bioactive secondary metabolite by the endophytic fungi (Soliman and Raizada 2013; Yenn et al. 2012). The anti-candidal activity of Phomopsis spp. isolated from the medicinal herb Orthosiphon stamineus has shown to be enhanced upon addition of aqueous host extract in the culture medium (Yenn et al. 2012). Some other studies have shown, however, that fungal endophytes could produce bioactive compounds in the absence of any plant tissues or extracts (Soliman et al. 2011).

The volatile profile of the endophytes that displayed the lowest ( $P$. canescens) and the highest (A. alternata) activity against yeasts revealed the presence of compounds previously reported to have antimicrobial activity against a variety of pathogens. For instances, 3-methyl-1-butanol was reported to have a high antimicrobial effect, and at low concentrations was shown to inhibit nearly $100 \%$ the growth of phytopathogens under in vitro conditions (Fialho et al. 2011). Phenylethyl alcohol is another organic compound identified in the present study and described to be a bacteriostatic agent that, at low concentrations, can inhibit the growth of Gram-negative bacteria, including Salmonella, Shigella, Aerobacter, Klebsiella, Escherichia, Pseudomonas and Proteus (Naz et al. 2013). Similarly, thujopsene has shown potent antibacterial activity, for example against phytopathogens (Manter et al. 2007), and $\beta$-cedrene was considered a powerful antimicrobial agent (Lin et al. 2012). Thus, this mixture of volatile compounds identified in the present study could participate in the microbial inhibition displayed by A. alternata as well as by $P$. canescens. This hypothesis is reinforced by the results obtained in the principal component analysis. In this analysis was found a clear relation between some VOCs produced by A. alternata and the inhibition displayed by this specie against the yeasts $C$. albicans and $C$. parapsilosis. Similarly, for 
the endophyte $P$. canescens was notice a relation between 1-octen-3-ol and E. coli, B. subtilis and P. aeruginosa inhibition. Although there was still need to confirm this hypothesis by performing for instances bioassays, the joint action of these VOCs seems to have potential utility in the treatment of infectious diseases caused by bacteria and yeasts. In fact, VOCs extracted from endophytic fungi have been reported to have antimicrobial effects against a variety of human and plant pathogens (Kudalkar et al. 2012; Mitchell et al. 2010).

We concluded that the endophytic fungus $P$. commune, $P$. canescens and A. alternata isolated from $O$. europaea possess antimicrobial agents capable to inhibit the tested Gram-positive, Gram-negative bacteria and yeasts. Alternaria alternata revealed high capacity to inhibit Candida spp. The role of host plant extracts was decisive over the antimicrobial potential, being observed different trends according to the microorganisms assayed. Volatile composition was influenced according to the tested endophytic fungus, being the most abundant components identified ascribed with antimicrobial potentialities. Therefore, future studies need to focus on the potential use of the endophytic fungus and their secondary metabolites (volatiles and nonvolatiles) as an alternative and innovative source of antimicrobial agents.

Acknowledgements This work is funded by FEDER funds through COMPETE (Programa Operacional Factores de Competitividade) and by national funds by FCT (Fundação para a Ciência e a Tecnologia) in the framework of the project "EndoBio-Isolation and screening of endophytic fungi for biological control of olive against Colletotrichum acutatum and Verticillium dahliae" (PTDC/ AGR-PRO/4354/2012)

\section{References}

Aly AH, Debbab A, Kjer J, Proksch P (2010) Fungal endophytes from higher plants: a prolific source of phytochemicals and other bioactive natural products. Fungal Divers 41:1-16

Banerjee D, Strobel G, Geary B, Sears J, Ezra D, Liarzi O, Coombs J (2010) Muscodor albus strain GBA, an endophytic fungus of Ginkgo biloba from United States of America, produces volatile antimicrobials. Mycology 1:179-186

Bertinetti BV, Peña NI, Cabrera GM (2009) An antifungal tetrapeptide from the culture of Penicillium canescens. Chem Biodivers 6:1178-1184

Carter GT (2011) Natural products and Pharma 2011: strategic changes spur new opportunities. Nat Prod Rep 28:1783-1789

Donadio S, Maffioli S, Monciardini P, Sosio M, Jabes D (2010) Antibiotic discovery in the twenty-first century: current trends and future perspectives. J Antibiot 63:423-430

Fernandes MRV, Costa e Silva TA, Pfenning LH, Costa-Neto CM, Heinrich TA, Alencar SM, Lima MA, Ikegaki M (2009) Biological activities of the fermentation extract of the endophytic fungus Alternaria alternata isolated from Coffea arabica L. Braz J Pharm Sci 45:677-685
Fialho MB, Moraes MHD, Tremocoldi AR, Pascholati SF (2011) Potential of antimicrobial volatile organic compounds to control Sclerotinia sclerotiorum in bean seeds. Pesq Agropec Bras 46:137-142

Gao SS, Li XM, Zhang Y, Li CS, Cui CM, Wang BG (2011) Comazaphilones A-F, azaphilone derivatives from the marine sediment-derived fungus Penicillium commune QSD-17. J Nat Prod $74: 256-261$

Gil-Chávez GJ, Villa JA, Ayala-Zavala JF, Heredia JB, Sepulveda D, Yahia EM, González-Aguilar GA (2013) Technologies for extraction and production of bioactive compounds to be used as nutraceuticals and food ingredients: an overview. Compr Rev Food Sci Food Saf 12:5-23

Guo BH, Wang YC, Zhou XW, Hu K, Tan F, Miao ZQ, Tang KX (2006) An endophytic taxol-producing fungus BT2 isolated from Taxus chinensis var. mairei. Afr J Biotechnol 5:875-877

Gutierrez RM, Gonzalez AM, Ramirez AM (2012) Compounds derived from endophytes: a review of phytochemistry and pharmacology. Curr Med Chem 19:2992-3030

Hellwig V, Grothe T, Mayer-Bartschmid A, Endermann R, Geschke FU, Henkel T, Stadler M (2002) Altersetin, a new antibiotic from cultures of endophytic Alternaria spp. Taxonomy, fermentation, isolation, structure elucidation and biological activities. J Antibiot 55:881-892

Högberg LD, Heddini A, Cars O (2010) The global need for effective antibiotics: challenges and recent advances. Trends Pharmacol Sci 31:509-515

Hyde KD, Soytong K (2008) The fungal endophyte dilemma. Fungal Divers 33:163-173

IDSA-Infectious Diseases Society of America (2010) The $10 \times$ '20 initiative: pursuing a global commitment to develop 10 new antibacterial drugs by 2020. Clin Infect Dis 50:1081-1083

Kjer J, Wray V, Edrada-Ebel R, Ebel R, Pretsch A, Lin W, Proksch P (2009) Xanalteric acids I and II and related phenolic compounds from an endophytic Alternaria sp. isolated from the mangrove plant Sonneratia alba. J Nat Prod 72:2053-2057

Kudalkar P, Strobel G, Riyaz-Ul-Hassan S, Geary B, Sears J (2012) Muscodor sutura, a novel endophytic fungus with volatile antibiotic activities. Mycoscience 53:319-325

Kusari S, Singh S, Jayabaskaran C (2014) Biotechnological potential of plant-associated endophytic fungi: hope versus hype. Trends Biotechnol 32:297-303

Laximanarayan R, Duse A, Wattal C et al (2013) Antibiotic resistance-the need for global solutions. Lancet Infect Dis 13:1057-1098

Lin J, Dou J, Xu J, Aisa HA (2012) Chemical composition, antimicrobial and antitumor activities of the essential oils and crude extracts of Euphorbia macrorrhiza. Molecules 17:5030-5039

Maheshwari R (2016) Fungi: experimental methods in biology, 2nd edn. CRC Press, New York

Manter DK, Kelsey RG, Karchesy JJ (2007) Antimicrobial activity of extractable conifer heartwood compounds toward Phytophthora ramorum. J Chem Ecol 33:2133-2147

Meirinhos J, Silva BM, Valentão P, Seabra RM, Pereira JA, Dias A, Andrade PB, Ferreres F (2005) Analysis and quantification of flavonoidic compounds from Portuguese olive (Olea europaea L.) leaf cultivars. Nat Prod Res 19:189-195

Mitchell AM, Strobel GA, Moore E, Robison R, Sears J (2010) Volatile antimicrobials from Muscodor crispans, a novel endophytic fungus. Microbiology 156:270-277

Moghaddam MH, Soltani J, Babolhavaeji F, Hamzei J, Nazeri S, Mirzaei S (2013) Bioactivities of endophytic Penicillia from Cupressaceae. J Crop Prot 2:421-433

Morath SU, Hung R, Bennett JW (2012) Fungal volatile organic compounds: a review with emphasis on their biotechnological potential. Fungal Biol Rev 26:73-83 
Mousa WK, Raizada MN (2013) The diversity of anti-microbial secondary metabolites produced by fungal endophytes: an interdisciplinary perspective. Front Microbiol 4:65

Musavi SF, Balakrishnan RM (2014) A study on the antimicrobial potentials of an endophytic fungus Fusarium oxysporum NFX 06. J Med Bioeng 3:162-166

Naz S, Cretenet M, Vernoux JP (2013) Current knowledge on antimicrobial metabolites produced from aromatic amino acid metabolism in fermented products. In: Méndez-Vilas A (ed) Microbial pathogens and strategies for combating them: science, technology and education. Formatex Research Center, Badajoz, pp 337-346

Nicoletti R, Fiorentino A, Scognamiglio M (2014) Endophytism of Penicillium species in woody plants. Open Mycol J 8:1-26

Pereira AP, Ferreira IC, Marcelino F, Valentão P, Andrade PB, Seabra R, Estevinho L, Bento A, Pereira JA (2007) Phenolic compounds and antimicrobial activity of olive (Olea europaea L. cv. Cobrançosa) leaves. Molecules 12:1153-1162

Pereira E, Santos A, Reis F, Tavares RM, Baptista P, Lino-Neto T, Almeida-Aguiar C (2013) A new effective assay to detect antimicrobial activity of filamentous fungi. Microbiol Res 168:1-5

Qadri M, Johri S, Shah BA, Khajuria A, Sidiq T, Lattoo SK, Abdin MZ, Riyaz-Ul-Hassan S (2013) Identification and bioactive potential of endophytic fungi isolated from selected plants of the Western Himalayas. SpringerPlus 2:8

Ramesha A, Sunitha VH, Srinivas C (2013) Antimicrobial activity of secondary metabolites from endophytic fungi isolated from Nerium oleander. Int J Pharm Biol Sci 4:683-693

Rios JL, Recio MC, Villar A (1988) Screening methods for natural products with antimicrobial activity: a review of the literature. J Ethnopharmacol 23:127-149

Rodriguez RJ, Woodward CJ, Redman RS (2012) Fungal influence on plant tolerance to stress. In: Southworth D (ed) Biocomplexity of plant-fungal interactions. Wiley-Blackwell, Oxford, pp 155-163
Sadananda TS, Nirupama R, Chaithra K, Govindappa M, Chandrappa CP, Vinay Raghavendra B (2011) Antimicrobial and antioxidant activities of endophytes from Tabebuia argentea and identification of anticancer agent (lapachol). JMPR 5:3643-3652

Selim KA, El-Beih AA, AbdEl-Rahman TM, El-Diwany AI (2012) Biology of endophytic fungi. Curr Res Environ Appl Mycol 2:31-82

Soliman SSM, Raizada MN (2013) Interactions between co-habitating fungi elicit synthesis of Taxol from an endophytic fungus in host Taxus plants. Front Microbiol 4:3

Soliman SS, Tsao R, Raizada MN (2011) Chemical inhibitors suggest endophytic fungal paclitaxel is derived from both mevalonate and non-mevalonate-like pathways. J Nat Prod 74:2497-2504

Strobel G (2006) Harnessing endophytes for industrial microbiology. Curr Opin Microbiol 9:240-244

Ul-Hassan SR, Strobel GA, Booth E, Knighton B, Flerchinger C, Sears J (2012) Modulation of volatile organic compound formation in the Mycodiesel-producing endophyte Hypoxylon sp. CI-4. Microbiology 158:465-473

Verma VC, Gond SK, Mishra A, Kumar A, Kharwar RN (2008) Selection of natural strains of fungal endophytes from Azadirachta indica A. Juss, with anti-microbial activity against dermatophytes. Curr Bioact Comp 4:36-40

Yang Y, Zhao H, Barrero RA et al (2014) Genome sequencing and analysis of the paclitaxel-producing endophytic fungus Penicillium aurantiogriseum NRRL 62431. BMC Genomics 15:69

Yenn TW, Lee CC, Ibrahim D, Zakaria L (2012) Enhancement of anti-candidal activity of endophytic fungus Phomopsis sp. ED2, isolated from Orthosiphon stamineus Benth, by incorporation of host plant extract in culture medium. J Microbiol 50:581-585

Yu H, Zhang L, Li L, Zheng C, Guo L, Li W, Sun P, Qin L (2010) Recent developments and future prospects of antimicrobial metabolites produced by endophytes. Microbiol Res 165:437-449 